\title{
Ethanolamine Oleate
}

National Cancer Institute

\section{Source}

National Cancer Institute. Ethanolamine Oleate. NCI Thesaurus. Code C61757.

A synthetic preparation of oleic acid and ethanolamine, a first generation

monoethanolamine with sclerotherapeutic activity. Ethanolamine oleate causes an acute, dose-related inflammatory reaction of the intimal endothelium of the vein. This leads to scarring at the inner wall of the veins and possible occlusion of the veins. The oleic acid component may also transiently activate coagulation and fibrinolytic systems through the release of tissue factor and activation of Hageman factor. 